\title{
Is it worthy to perform elective total pancreatectomy considering morbidity or mortality?: An experience from a high-volume center
}

\author{
Ji Hye JUNG, So Kyung YOON, So Jeong YOON, Sang Hyun SHIN, Jin Seok HEO, In Woong HAN* \\ Division of Hepatobiliary-Pancreatic Surgery, Department of Surgery, Samsung Medical Center, \\ Sungkyunkwan University School of Medicine, Seoul, Korea
}

Introduction: Total pancreatectomy (TP) is mostly performed for diseases involving the entire pancreas including various pathology. However, there is still a reluctance to perform TP due to high postoperative morbidity or mortality, and life-long endocrine and exocrine pancreatic insufficiency. This retrospective study aimed to evaluate postoperative outcomes in a high-volume center and identify the risk factors affecting major morbidity and mortality after TP.

Methods: From 1995 to 2015, a total of 142 patients who underwent elective TP at Samsung Medical Center were included in this study. One-stage TP was defined as elective primary TP, and in whom an intraoperative decision to extend the planned resection to TP, whereas 2-stage TP was elective completion TP due to recurred tumor. Patients who underwent TP in an emergency setting were excluded. Postoperative mobidity or pancreatectomy-specific complication was defined according to Clavien-Dindo classification (CDC) or ISGPF classification.

Results: There were no statistically significant differences between 1-stage TP $(\mathrm{n}=128)$ and 2-stage TP $(\mathrm{n}=14)$ in clinical, operative, pathologic variables. Overall major morbidity more than CDC $\geq 3$ or ISGPF grade B/C were occurred in 25 patients (17.6\%). The readmission rate within 90 -day including DM control was $20.4 \%$. There was no in-hospital mortality among all enrolled patients. Multiple underlying diseases (OR, 3.350; 95\% CI, 1.244-9.019; $p=0.017)$ and longer operative time (OR, $1.005 ; 95 \%$ CI, $1.000-1.010 ; p=0.041)$ were identified an independent risk factors for major morbidity after multivariable analysis.

Conclusions: TP are safe and feasible procedures with satisfactory early surgical outcomes when performed at high-volume center. 